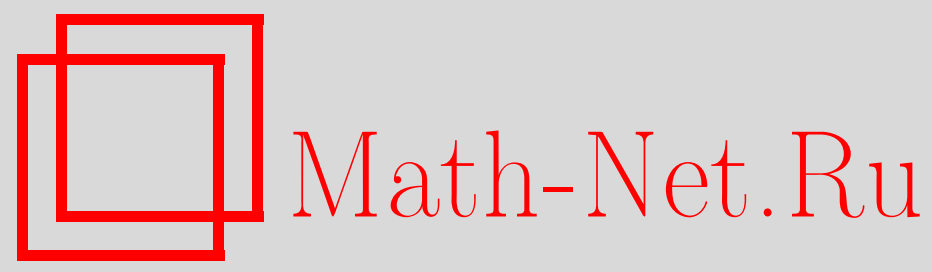

Р. О. Гринив, А. А. Шкаликов, Экспоненциальная устойчивость полугрупп, связанных с некоторыми операторными моделями в механике, Матем. заметки, 2003, том 73, выпуск 5, 657-664

DOI: https://doi.org/10.4213/mzm217

Использование Общероссийского математического портала Math-Net.Ru подразумевает, что вы прочитали и согласны с пользовательским соглашением http://www . mathnet.ru/rus/agreement

Параметры загрузки:

IP: 54.198 .67 .100

26 апреля 2023 г., $17: 29: 45$

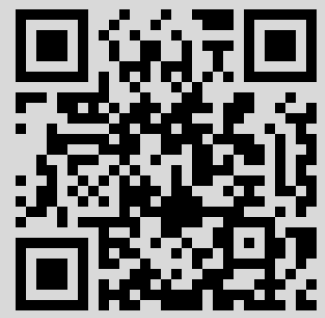




\title{
ЭКСПОНЕНЦИАЛЬНАЯ УСТОЙЧИВОСТЬ ПОЛУГРУПП, СВЯЗАННЫХ С НЕКОТОРЫМИ ОПЕРАТОРНЫМИ МОДЕЛЯМИ В МЕХАНИКЕ
}

\author{
Р. О. Гринив, А.А. Шкаликов
}

Рассматриваются уравнения вида $\ddot{x}+B \dot{x}+A x=0$, где $x=x(t)$ - функция со значениями в гильбертовом пространстве $\mathscr{H}$, оператор $B$ симметрический, а $A$-равномерно положительный и самосопряженный в $\mathscr{H}$. С этим уравнением ассоциируется линейный оператор $\mathscr{T}$, который генерирует $C_{0}$-полугруппу в энергетическом пространстве $\mathscr{H}_{1} \times \mathscr{H}$. Показано, что эта полугруппа является экспоненциально устойчивой, если оператор $B$ равномерно положителен и подчинен оператору $A$ в смысле квадратичных форм.

Библиографоия: 20 названий.

1. Введение. Линеаризованные уравнения, появляющиеся в различных задачах теории упругости и гидромеханики, часто записываются в виде

$$
\ddot{x}+B \dot{x}+A x=0 .
$$

Здесь $x(t)$ - функция со значениями в гильбертовом пространстве $\mathscr{H}$, а $A$ и $B$ - некоторые (неограниченные) операторы в $\mathscr{H}$. Обычно (см. [1], [2]) оператор $A$ самосопряжен и равномерно положителен (отвечает за потенциальную энергию системы), а оператор $B$ симметричен и неотрицателен (отвечает за силы демпфирования).

Энергия решения $x(t)$ уравнения (1) определяется функционалом

$$
E(x(t)):=\frac{1}{2}\left(\|\dot{x}\|^{2}+\left\|A^{1 / 2} x\right\|^{2}\right) .
$$

Из уравнения (1) находим, что при $B \geqslant 0$ энергия системы убьвает со временем, ибо

$$
\frac{d}{d t} E(x(t))=-(B \dot{x}(t), \dot{x}(t)) \leqslant 0 .
$$

Для многих конкретных задач механики удается показать, что энергия убывает экспоненциально. Задаче об описании абстрактных моделей, для которых наблюдается это

Работа выполнена при поддержке Российского фонда фундаментальных исследований, гранты № 00-15-96100 и № 01-01-00691. 
явление, в литературе уделено большое внимание (см., например, работы [1]-[6] и имеюшиеся там ссылки). Настоящая заметка посвящена этой же проблеме. Здесь мы даем относительно простое доказательство экспоненциального убывания энергии при значительно более слабых условиях на операторы $A$ и $B$, нежели имевшиеся ранее.

Обозначим через $\mathscr{H}_{\theta}, \theta \in \mathbb{R}$, шкалу гильбертовых пространств, порожденньх равномерно положительным самосопряженньм оператором $A$. Таким образом, при $\theta>0$ пространство $\mathscr{H}_{\theta}$ совпадает с областью определения $\mathfrak{D}\left(A^{\theta / 2}\right)$ оператора $A^{\theta / 2}$ и наделяется нормой $\|x\|_{\theta}:=\left\|A^{\theta / 2} x\right\|$, а при $\theta<0$ это пространство совпадает с пополнением исходного пространства $\mathscr{H}$ по такой же норме. Уравнение (1) можно переписать в виде

$$
\dot{\mathbf{x}}(t)=\mathscr{T}_{0} \mathbf{x}(t),
$$

где $\mathbf{x}(t)=\left(\begin{array}{l}x_{1}(t) \\ x_{2}(t)\end{array}\right)$ функция со значениями в энергетическом пространстве $\mathscr{E}:=$ $\mathscr{H}_{1} \mathscr{H}$, и

$$
\mathscr{T}_{0}:=\left(\begin{array}{cc}
0 & I \\
-A & -B
\end{array}\right), \quad \mathfrak{D}\left(\mathscr{T}_{0}\right)=\left\{\left(\begin{array}{l}
x_{1} \\
x_{2}
\end{array}\right), \quad x_{1} \in \mathfrak{D}(A), \quad x_{2} \in \mathfrak{D}(B)\right\}
$$

Легко видеть, что $\mathbf{x}(t)$ является решением уравнения (2) в том и только том случае, когда $x_{1}(t)$ есть решение $(1)$ и $x_{2}(t)=\dot{x}_{1}(t)$, причем тогда $\|\mathbf{x}\|_{\mathscr{E}}^{2}=2 E\left(x_{1}\right)$. Известно (см. [6]), что в случае, когда $B \geqslant 0$ и $B$ подчинен оператору $A$ в обычном смысле, т.е. когда

$$
\mathfrak{D}(B) \supset \mathfrak{D}(A),
$$

оператор $\mathscr{T}_{0}$ допускает замькание $\mathscr{T}$, причем $\mathscr{T}$ есть максимально диссипативньй оператор в пространстве $\mathscr{E}$. Следовательно, $\mathscr{T}$ генерирует $C_{0}$-полугруппу сжатий $\exp (t \mathscr{T})$ в $\mathscr{H}$, и любое решение уравнения $(2)$ имеет вид $\mathbf{x}(t)=\exp (t \mathscr{T}) \mathbf{x}_{0}$, где $\mathbf{x}_{0}=\mathbf{x}(0)-$ начальное состояние. Поэтому $E(x)$ экспоненциально убьвает для любого решения уравнения (1) в том и только том случае, когда полугруппа $\exp (t \mathscr{T})$ является экспоненииально устойчивой, т.е. когда әкспоненциальный тип $\omega(\mathscr{T}) C_{0}$-полугруппы $\exp (t \mathscr{T})$ (см. [7], [8]), определяемьй равенством

$$
\omega(\mathscr{T}):=\limsup _{t \rightarrow \infty} \frac{1}{t}\|\exp (t \mathscr{T})\|_{\mathscr{E}}
$$

является отрицательным.

В работах [1]-[3], [5] было показано, что $\omega(\mathscr{T})<0$, если, помимо (4), вьполнено одно из следующих условий:

а) $\|B x\| \geqslant \varepsilon\left\|A^{1 / 2} x\right\|$ для всех $x \in \mathfrak{D}\left(A^{1 / 2}\right)$ при некотором $\varepsilon>0$;

б) оператор $B$ сравним с $A^{\alpha}$ при $\alpha \in[1 / 2,1]$, т.е.

$$
c_{1}\left(A^{\alpha} x, x\right) \leqslant(B x, x) \leqslant c_{2}\left(A^{\alpha} x, x\right) \quad \text { для всех } x \in \mathfrak{D}\left(A^{\alpha / 2}\right), \quad c_{1}, c_{2}>0 .
$$


В действительности при таких предположениях доказано большее: полугруппа $\exp (t \mathscr{T})$ является аналитической, и тогда экспоненциальньй тип $\omega(\mathscr{T})$ совпадает со спектральной абсииссой

$$
s(\mathscr{T}):=\sup \{\operatorname{Re} \lambda \mid \lambda \in \sigma(\mathscr{T})\},
$$

где $\sigma(\mathscr{T})$ - спектр оператора $\mathscr{T}$. (Отметим, что всегда вьполнено неравенство $s(\mathscr{T}) \leqslant$ $\omega(\mathscr{T})$.$) В [4] показано, что совпадение s(\mathscr{T})$ и $\omega(\mathscr{T})$ имеет место также тогда, когда $B$ сравним с $A^{\alpha}$ при любом $\alpha \in(0,1 / 2)$, однако полугруппа в этом случае не аналитическая, а принадлежит классу Жеврея с показателем $1 / 2 \alpha$. В работе [6] задача об аналитичности полугруппы $\exp (t \mathscr{T})$ решалась для секториальных операторов $B$.

В этой заметке мы отказываемся от условия (4) (есть конкретные задачи, где оно не выполняется) и заменяем его более слабым условием подчиненности оператора $B$ оператору $A$ в смысле квадратичных форм. Последнее означает, что линеал $\mathfrak{D}(B) \cap \mathscr{H}_{1}$ плотен в пространстве $\mathscr{H}_{1}=\mathfrak{D}\left(A^{1 / 2}\right)$, и справедлива оценка

$$
|(B x, x)| \leqslant c\left(A^{1 / 2} x, A^{1 / 2} x\right) \quad \text { для всех } \quad x \in \mathfrak{D}(B) \cap \mathscr{H}_{1},
$$

где $c>0$ - постоянная. Это условие эквивалентно тому, что оператор $B$ продолжается по непрерывности как ограниченный оператор из $\mathscr{H}_{1}$ в $\mathscr{H}_{-1}$. Действительно, из оценки (5) следует, что оператор $K=A^{-1 / 2} B A^{-1 / 2}$ плотно определен в $\mathscr{H}$, а его числовой образ ограничен. Следовательно, $K$ продолжается по непрерьвности как ограниченный оператор из $\mathscr{H}_{1}$ в $\mathscr{H}_{-1}$. Обратное утверждение очевидно. Отметим также, что в случае $B \geqslant 0$ условие подчиненности в смысле квадратичных форм эквивалентно включению $\mathfrak{D}\left(B_{F}^{1 / 2}\right) \supset \mathfrak{D}\left(A^{1 / 2}\right)$, где $B_{F}$ - расширение по Фридрихсу оператора $B$ (см. [6]).

Поясним, почему условие (5) является более слабым, нежели (4). Известно (см., например, [9]), что (4) влечет ограниченность оператора $B: \mathscr{H}_{2} \rightarrow \mathscr{H}$, а потому и оператора $B^{*}: \mathscr{H}_{-2} \rightarrow \mathscr{H}$. С учетом условия симметричности $B \subset B^{*}$, из интерполяционной теоремы (см., например, [10, гл. I]) получаем, что оператор $B: \mathscr{H}_{1} \rightarrow \mathscr{H}_{-1}$ также ограничен.

Наша дальнейшая цель - показать, что полугруппа $\exp (t \mathscr{T})$ корректно определена, если оператор $B \geqslant 0$ подчинен оператору $A$ в смысле квадратичных форм, причем условие $B \gg 0$ достаточно для того, чтобы ее экспоненциальньй тип $\omega(\mathscr{T})$ был отрицателен. Более того, как показано в замечании 1 , эти условия в некотором смысле являются также необходимыми для экспоненщиальной устойчивости. Конечно, условие $B \gg 0$ не гарантирует аналитичность полугруппы, и мы не можем утверждать, что $\omega(\mathscr{T})$ совпадает со спектральной абсциссой $s(\mathscr{T})$. Однако для числа $\omega(\mathscr{T})$ мы получим эффективную оценку.

2. Формулировка теоремы. Далее, как обычно, через $(x, y)$ обозначаем скалярное произведение элементов $x, y \in \mathscr{H}$, и такое же обозначение сохраняем для значения функционала $x \in \mathscr{H}_{-1}$ на элементе $y \in \mathscr{H}_{1}$.

С уравнением (1) свяжем его символ

$$
L(\lambda)=\lambda^{2}+\lambda B+A \text {. }
$$

Условие (5) не гарантирует, что линеал $\mathscr{L}=\mathfrak{D}(B) \cap \mathfrak{D}(A)$ плотен в $\mathscr{H}$ (может даже оказаться, что $\mathscr{L}=\{0\})$, поэтому оператор-функция $L(\lambda)$, вообще говоря, не определена в пространстве $\mathscr{H}$. Однако $A$ допускает изометрическое продолжение как оператор из 
$\mathscr{H}_{1}$ в $\mathscr{H}_{-1}$, а условие $(5)$ влечет, что $B$ имеет ограниченное продолжение из $\mathscr{H}_{1}$ в $\mathscr{H}_{-1}$. Обозначим эти продолжения через $\tilde{A}$ и $\widetilde{B}$, соответственно. Тогда оператор

$$
\tilde{L}(\lambda)=\lambda^{2}+\lambda \widetilde{B}+\tilde{A}
$$

корректно определен при $\lambda \in \mathbb{C}$ как ограниченньй оператор из $\mathscr{H}_{1}$ в $\mathscr{H}_{-1}$, или как неограниченньй оператор в $\mathscr{H}_{-1}$ с областью $\mathfrak{D}(\tilde{L})=\mathscr{H}_{1}$. Теперь можно определить и оператор $L(\lambda)$ в пространстве $\mathscr{H}$, положив $L(\lambda) x=\tilde{L}(\lambda) x$ на области

$$
\mathfrak{D}(L(\lambda))=\left\{x \in \mathscr{H}_{1} \mid \tilde{L}(\lambda) x \in \mathscr{H}\right\} .
$$

Конечно, $\mathfrak{D}(L(\lambda))$ может зависеть от $\lambda$. Согласно [11] множество значений $\lambda \in \mathbb{C}$, для которых оператор $L(\lambda)$ (оператор $\tilde{L}(\lambda))$ не имеет ограниченного обратного в $\mathscr{H}\left(\right.$ в $\left.\mathscr{H}_{-1}\right)$, назовем спектром пучка $L(\lambda)$ (обобщенным спектром $L(\lambda))$ и будем обозначать его $\sigma(L)$ (соответственно $\tilde{\sigma}(L))$.

Для формулировки теоремы определим числа

$$
\begin{gathered}
\beta:=\inf \{(B x, x) \mid x \in \mathfrak{D}(B),\|x\|=1\}, \\
r(L):=\inf \{r \in \mathbb{R} \mid \tilde{L}(\lambda) \succ A \quad \text { для всех } \lambda>r\},
\end{gathered}
$$

где $\tilde{L}(\lambda) \succ A$ означает, что

$$
(\tilde{L}(\lambda) x, x) \geqslant \varepsilon(A x, x)
$$

при некотором $\varepsilon>0$ (зависящем от $\lambda$ ). Очевидно, из условия $(5)$ следует $r(L)<0$.

Teорема. Пусть $A=A^{*} \gg 0, B \geqslant 0$ u $B$ подчинен $A$ в смысле квадратичных форм. Тогда оператор $\mathscr{T}_{0}$, определенный равенством (3), является существенно m-диссипативным т.е. его замыкание $\mathscr{T}$ является $m$-диссипативным оператором в энергетическом пространстве $\mathscr{E}=\mathscr{H}_{1} \times \mathscr{H}$. Оператор $\mathscr{T}$ определяется равенством

$$
\mathscr{T}\left(\begin{array}{l}
x_{1} \\
x_{2}
\end{array}\right)=\left(\begin{array}{c}
x_{2} \\
-\tilde{A} x_{1}-\widetilde{B} x_{2}
\end{array}\right)
$$

на области

$$
\mathfrak{D}(\mathscr{T})=\left\{\left(\begin{array}{l}
x_{1} \\
x_{2}
\end{array}\right) \mid x_{1}, x_{2} \in \mathscr{H}_{1}, \quad \tilde{A} x_{1}+\widetilde{B} x_{2} \in \mathscr{H}\right\} .
$$

Cпектр оператора $\mathscr{T}$ содержится в $\tilde{\sigma}(L)$ - обобщенном спектре пучка $L(\lambda)$. При $\lambda \notin \tilde{\sigma}(L)$ и $\lambda \neq 0$ резольвента $(\mathscr{T}-\lambda)^{-1}$ имеет представление

$$
(\mathscr{T}-\lambda)^{-1}=\left(\begin{array}{cc}
\lambda^{-1}\left(\tilde{L}^{-1}(\lambda) \tilde{A}-I\right) & -\tilde{L}^{-1}(\lambda) \\
\tilde{L}^{-1}(\lambda) \tilde{A} & -\lambda \tilde{L}^{-1}(\lambda)
\end{array}\right) .
$$

Экспоненциальный тип $\omega(\mathscr{T})$ полугруппы $\exp (t \mathscr{T})$ допускает оценку

$$
\omega(\mathscr{T}) \leqslant \omega_{0}:=\max \left\{r(L),-\frac{\beta}{2}\right\} .
$$

В частности, если $B \gg 0$, то $\omega(\mathscr{T})<0$, m.е. полугруппа $\exp (t \mathscr{T})$ әкспоненииально устойчива. 
3. Предварительные результаты. Имеется обширная литература о необходимых и достаточных условиях на операторы в гильбертовом или банаховом пространствах, чтобы они были генераторами равномерно устойчивых $C_{0}$-полугрупп (см., например, [8], [12]-[19] и имеюшиеся там ссылки). Мы воспользуемся результатом Герхарта [13] для случая гильбертова пространства. Напомним, что пространство Харди $H^{\infty}(\alpha)$ в полуплоскости $\Pi(\alpha):=\{z \in \mathbb{C} \mid \operatorname{Re} z>\alpha\}$ состоит из голоморфных равномерно ограниченных функций в П $(\alpha)$. Это же обозначение мы сохраняем и для пространств голоморфных равномерно ограниченных оператор-функций в $(\alpha)$.

ПРЕДЛОЖЕНИЕ 1 (см. [13]). Пусть $T$ - генератор $C_{0}$-полугруппь $S(t)$ в гильбертовом пространстве $\mathscr{H}$. Тогда ее әкспоненииальный тип определяется равенством

$$
\omega(T)=\inf \left\{\alpha \in \mathbb{R} \mid(T-\lambda)^{-1} \in H^{\infty}(\alpha)\right\} .
$$

Далее используются понятия замкнутой квадратичной формы и ассоциированного с ней оператора. Необходимые сведения об этих понятиях можно найти в книге [20,гл. VI].

ПРЕДЛОЖЕНИЕ 2. Пусть $\lambda \in(r(L), \infty)$. Тогда в условиях теоремы квадратичная форма $\mathfrak{l}_{\lambda}[x]:=(\tilde{L}(\lambda) x, x)$, определенная на әлементах $x \in \mathscr{H}_{1}$, замкнута, $u$ ассочиированный с ней согласно первой теореме о представлении оператор совпадает с $L(\lambda)$. Более того, оператор $L(\lambda)$ самосопряжен, равномерно положителен, причем $\mathfrak{D}\left([L(\lambda)]^{1 / 2}\right)=\mathscr{H}_{1}$, а оператор $\tilde{L}(\lambda)$ осуществляет биекиию $\mathscr{H}_{1}$ на $\mathscr{H}_{-1}$.

ДокАЗАТЕЛЬСтво. Допустим на время, что замкнутость квадратичной формы $\mathfrak{l}_{\lambda}$ уже установлена. Поскольку при $\lambda>r(L)$ квадратичная форма $\mathfrak{l}_{\lambda}$ равномерно положительна, по первой теореме о представлении ей соответствует равномерно положительный самосопряженньй оператор $L_{\lambda}$ в $\mathscr{H}$ такой, что $\mathfrak{D}\left(L_{\lambda}^{1 / 2}\right)=\mathscr{H}_{1}$. Для $x \in \mathfrak{D}\left(L_{\lambda}\right)$ и произвольного $y \in \mathscr{H}_{1}$ имеем

$$
\left(L_{\lambda} x, y\right)=\mathfrak{l}_{\lambda}[x, y]=(\tilde{L}(\lambda) x, y)
$$

поэтому $L_{\lambda} x=\tilde{L}(\lambda) x=L(\lambda) x$. Таким образом, $L(\lambda)$ есть симметрическое расширение $L_{\lambda}$, и поэтому $L_{\lambda}=L(\lambda)$. Далее, оператор $L_{\lambda}^{1 / 2}$ отображает биективно $\mathscr{H}_{1}$ на $\mathscr{H}$, а его сопряженный $\left(L_{\lambda}^{1 / 2}\right) *$ (являюшийся расширением $L_{\lambda}^{1 / 2}$ ) реализует биекцию $\mathscr{H}$ в $\mathscr{H}_{-1}=\left(\mathscr{H}_{1}\right)^{*}$. Следовательно, $\tilde{L}(\lambda)=\left(L_{\lambda}^{1 / 2}\right)^{*} L_{\lambda}^{1 / 2}-$ биекция $\mathscr{H}_{1}$ на $\mathscr{H}_{-1}$.

Итак, осталось доказать замкнутость квадратичной формы $\mathfrak{l}_{\lambda}$ на $\mathscr{H}_{1}$. При $\lambda=0$ это очевидно. Из теоремы о замкнутости суммы форм [20, гл. VI, теорема 1.33] и условия (5) получаем, что форма $\mathfrak{l}_{\lambda}$ замкнута в $\mathscr{H}_{1}$, если $\lambda$ принадлежит некоторой окрестности нуля. Пусть $(a, b)$ - наибольший интервал такой, что квадратичные формы $\mathfrak{l}_{\lambda}$ замкнуты на $\mathscr{H}_{1}$ при всех $\lambda \in(a, \infty)$. Предположим, что $a>r(L)$. Согласно определению

$$
\mathfrak{l}_{a}[x]=(\tilde{L}(a) x, x) \geqslant \varepsilon(A x, x), \quad x \in \mathscr{H}_{1},
$$

при некотором $\varepsilon>0$. Пусть $c$ - константа из (5). Вновь используя теорему о замкнутости суммы форм, получаем, что квадратические формы $\mathfrak{l}_{a}$ и $\mathfrak{l}_{a+\delta}$ с произвольным $\delta \in(-\varepsilon / 2 c, \varepsilon / 2 c)$ замкнуты или не замкнуты на $\mathscr{H}_{1}$ одновременно. Поскольку $\mathfrak{l}_{a+\delta}$ замкнута на $\mathscr{H}_{1}$ при всех $\delta>0$, мы получаем противоречие с выбором $a$. Поэтому предположение $a>r(L)$ неверно, т.е. $a \leqslant r(L)$. Аналогично доказьвается, что $b=\infty$. 
4. Доказательство теоремы. Разобьем его на несколько шагов.

Шаг 1. Условие диссипативности

$$
\operatorname{Re}\left(\mathscr{T}_{0} x, x\right)=-\left(B x_{2}, x_{2}\right), \quad x=\left(x_{1}, x_{2}\right)^{T}
$$

проверяется непосредственно. Следовательно, $\mathscr{T}_{0}$ допускает замыкание $\mathscr{T}$. Согласно определению $\mathscr{T}_{0}$ является существенно $m$-диссипативным, если образ $\mathscr{T}_{0}-\lambda$ плотен в $\mathscr{E}$ при некотором (а тогда при всех) $\lambda$ из правой полуплоскости. Покажем, что при любых $y_{1} \in \mathfrak{D}(B), y_{2} \in \mathscr{H}, \lambda>0$ уравнение

$$
\left(\mathscr{T}_{0}-\lambda\right)\left(x_{1}, x_{2}\right)^{T}=\left(y_{1}, y_{2}\right)^{T}
$$

разрешимо. Согласно предложению 2 при $\lambda>0$ оператор $L(\lambda)$ обратим, а тогда решение этого уравнения находим явно:

$$
x_{1}=-L^{-1}(\lambda)\left(y_{2}+(B+\lambda) y_{1}\right), \quad x_{2}=\lambda x_{1}+y_{1} .
$$

Поскольку $\mathfrak{D}(B)$ плотно в $\mathscr{H}_{1}$, то образ $\mathscr{T}_{0}-\lambda$ плотен в $\mathscr{E}=\mathscr{H}_{1} \times \mathscr{H}$.

Далее, оператор $\mathscr{T}$, определенньй равенством (6) на области (7), является диссипативным расширением $\mathscr{T}_{0}$. Так как $\overline{\mathscr{T}_{0}}$ не допускает нетривиальных диссипативных расширений в $\mathscr{E}$, равенство $\mathscr{T}=\overline{\mathscr{T}_{0}}$ вытекает из замкнутости $\mathscr{T}$. Замкнутость $\mathscr{T}$ устанавливается непосредственно из определения, точно так же, как в теореме 1 работы [6].

Шаг 2. Заметим, что $\mathscr{T}$ ограниченно обратим в $\mathscr{E}$, причем

$$
\mathscr{T}^{-1}=\left(\begin{array}{cc}
-\tilde{A}^{-1} \widetilde{B} & -\tilde{A}^{-1} \\
I & 0
\end{array}\right) .
$$

Далее, при $\lambda \neq 0, \lambda \notin \tilde{\sigma}(L)$, можно явно вьгислить резольвенту $(\mathscr{T}-\lambda)^{-1}$, которая выражается формулой (8). Из этого представления и предложения 1 получаем следующий результат.

ПРЕДЛОЖЕНИЕ 3. $C_{0}$-полугруппа $\exp (t \mathscr{T})$ имеет әкспоненциальный тuп $\omega(\mathscr{T}) \leqslant$ $\omega_{0}$ в том и только том случае, если при любом $\alpha>\omega_{0}$ все четыре оператор-функuuu

$$
\begin{array}{cc}
f_{1}(\lambda):=\left(\lambda-\omega_{0}\right)^{-1} A^{1 / 2} \tilde{L}^{-1}(\lambda) \tilde{A}^{1 / 2}, \quad & f_{2}(\lambda):=A^{1 / 2} \tilde{L}^{-1}(\lambda), \\
f_{3}(\lambda):=\tilde{L}^{-1}(\lambda) \tilde{A}^{1 / 2}, \quad & f_{4}(\lambda):=\lambda \tilde{L}^{-1}(\lambda)
\end{array}
$$

принадлежат пространству $H^{\infty}(\alpha)$ в равномерной операторной топологии пространства $\mathscr{H}$. Здесь $\tilde{A}^{1 / 2}=\tilde{A} A^{-1 / 2}$ - расширение $A^{1 / 2}$ как оператора из $\mathscr{H}$ в $\mathscr{H}_{-1}$.

Шаг 3. Фиксируем произвольное число $\delta$, подчиненное условию $\delta>\omega_{0}:=\max \{-\beta / 2$, $r(L)\}$. Положим $B_{1}=B+2 \delta, A_{1}=L(\delta)$. Через $\widetilde{B}_{1}, \tilde{A}_{1}$ обозначим соответствующие расширения $B_{1}$ и $A_{1}$ как операторов из $\mathscr{H}_{1}$ в $\mathscr{H}_{-1}$. Рассмотрим оператор-функцию

$$
\tilde{L}_{1}(\lambda):=\lambda^{2}+\lambda \widetilde{B}_{1}+\tilde{A}_{1}=\tilde{L}(\lambda+\delta)
$$

из $\mathscr{H}_{1}$ в $\mathscr{H}_{-1}$ и соответствующую ей оператор-функцию $L_{1}(\lambda)=L(\lambda+\delta)$ в $\mathscr{H}$. Согласно предложению 2 имеем $A_{1} \gg 0, \mathfrak{D}\left(A_{1}\right)=\mathscr{H}_{1}$. Очевидно, $B_{1}$ подчинен $A_{1}$ в смысле квадратичных форм, и из определения числа $\beta$ следует $B_{1}>0$. 
Рассмотрим оператор

$$
\mathscr{T}_{1}=\left(\begin{array}{cc}
0 & I \\
-A_{1} & B_{1}
\end{array}\right)
$$

По доказанному на шаге $1 \mathscr{T}_{1}$ сушественно $m$-диссипативен в $\mathscr{E}$, а его замыкание $\overline{\mathscr{T}_{1}}$ генерирует $C_{0}$-полугрупп сжатий в $\mathscr{E}$. Из предложения 3 получаем, что все четыре функции

$$
\begin{array}{lll}
g_{1}(\lambda):=\left(\lambda-\omega_{0}\right)^{-1} A_{1}^{1 / 2} \tilde{L}_{1}^{-1}(\lambda) \tilde{A}_{1}^{1 / 2}, & g_{2}(\lambda):=A_{1}^{1 / 2} \tilde{L}_{1}^{-1}(\lambda) \\
g_{3}(\lambda):=\tilde{L}_{1}^{-1}(\lambda) \tilde{A}_{1}^{1 / 2}, & g_{4}(\lambda):=\lambda \tilde{L}_{1}^{-1}(\lambda)
\end{array}
$$

принадлежат пространствам Харди $H^{\infty}(\alpha)$ при любом $\alpha>0$. Так как оба оператора $A^{1 / 2}$ и $A_{1}^{1 / 2}$ осуществляют биекцию $\mathscr{H}_{1}$ на $\mathscr{H}$, то оператор $K=A_{1}^{1 / 2} A^{-1 / 2}$ ограничен и обратим в $\mathscr{H}$. Учитывая равенства

$$
A_{1}^{1 / 2}=K A^{1 / 2}, \quad \tilde{A}_{1}^{1 / 2}=\tilde{A}^{1 / 2} K^{*}, \quad \tilde{L}_{1}(\lambda)=\tilde{L}(\lambda+\delta),
$$

получаем, что все четыре функции в (10) принадлежат пространствам Харди $H^{\infty}(\alpha)$ при любом $\alpha>\delta$. Вновь воспользовавшись предложением 3 , получим, что полугруппа $\exp (t \mathscr{T})$ имеет экспоненциальньй тип $\leqslant \delta$. Это завершает доказательство теоремы.

ЗАмЕчАниЕ 1 . В заключение отметим, что для симметрического оператора $B$ условия (5) и $B \gg 0$ являются в некотором смысле необходимыми для экспоненциальной устойчивости полугруппы $\exp (t \mathscr{T})$. Действительно, при нарушении $(5)$ оператор $\mathscr{T}$ не является ограниченно обратимьм в $\mathscr{E}($ ср. шаг 2 доказательства теоремы), т.е. $0 \in \sigma(\mathscr{T})$ и $\omega(\mathscr{T}) \geqslant s(\mathscr{T})=0$. Если же не требовать равномерной положительности оператора $B$, то, например, при $B=A^{-1}$ также получим $\omega(\mathscr{T}) \geqslant s(\mathscr{T})=0$.

\section{СПИСОК ЦИТИРОВАННОЙ ЛИТЕРАТУРЫ}

[1] Chen G., Russel D. L. A mathematical model for linear elastic systems with structural damping // Quart. Appl. Math. 1982. №1. P. 433-454.

[2] Chen S., Triggiani R. Proof of extensions of two conjectures on structural damping for elastic systems // Pacific J. Math. 1989. V. 136. № 1. P. 15-55.

[3] Huang F. On the mathematical model for linear elastic systems with analytic damping // SIAM J. Control Optim. 1988. V. 26. № 3. P. 714-724.

[4] Chen S., Triggiani R. Gevrey class semigroups arising from elastic systems with gentle dissipation: the case $0<\alpha<1 / 2$ // Proc. Amer. Math. Soc. 1990. V. 110. № 2. P. 401-415.

[5] Huang F. Some problems for linear elastic systems with damping // Acta Math. Sci. 1990. V. 10. № 3. P. 316-326.

[6] Гринив Р. О., Шкаликов А. А. Операторные модели в теории упругости и гидромеханике и ассоциированные с ними аналитические полугруппы // Вестн. Моск. ун-та. Сер. матем., мex. 1999. № 5. С. 5-14.

[7] Любич Ю.И. Классическое и локальное преобразование Лапласа в абстрактной задаче Коши // УМН. 1966. Т. 21(129). №3. С. 3-51.

[8] Pazy A. Semigroups of Linear Operators and Applications to Partial Differential Equations // Applied Mathematical Sciences. V. 44. New York-Berlin: Springer-Verlag, 1983.

[9] Kato T. A generalization of the Heinz inequality // Proc. Japan. Acad. 1961. V. 37. P. 305-308.

[10] Лионс ЖК.-Л., Мадженес Е. Неоднородные граничные задачи и приложения. І. М.: Мир, 1971. 
[11] Shkalikov A. A. Operator pencils arising in elasticity and hydrodynamics: the instability index formula // Operator Theory: Adv. and Appl. V. 87: Birkhäuser-Verlag, 1996. P. 358-385.

[12] Datko R. Extending a theorem of A. M. Liapunov to Hilbert space // J. Math. Anal. Appl. 1970. V. 32. P. 610-616.

[13] Gearhart L. Spectral theory for contraction semigroups on Hilbert spaces // Trans. Amer. Math. Soc. 1978. V. 236. P. 385-394.

[14] Van Casteren J. A. Operators similar to unitary or self-adjoint ones // Pacific J. Math. 1983. V. 104. № 1. P. 241-255.

[15] Набоко С. Н. Об условии подобия унитарным или самосопряженньм операторам // Функцион. анал. и его прилож. 1984. Т. 18. №1. С. 16-27.

[16] Маламуд М.M. Критерий подобия замкнутого оператора самосопряженному // Укр. матем. ж. 1985. Т. 37. №1. С. 49-56.

[17] Rolewicz S. On uniform $N$-equistability // J. Math. Anal. Appl. 1986. V. 115. P. 431-441.

[18] Littman W. A Generalization of a Theorem of Datko and Pazy // Lecture Notes in Control and Inform. Sci. V. 130. Berlin-New York: Springer, 1989. P. 318-323.

[19] Гомилко А. М. Об условиях на производящий оператор равномерно ограниченной $C_{0}$-полугруппы операторов // Функцион. анализ и его прилож. 2000. Т. 33. № 4. С. 66-69.

[20] Като Т. Теория возмущений линейных операторов. М.: Мир, 1972.

Институт прикладных проблем механики и математики,

Поступило

Львов, Украина

28.10 .2002

Московский государственный университет им. М.В. Ломоносова

E-mail : rhryniv@iapmm.lviv.ua

ashkalikov@yahoo.com 